\title{
Actitudes hacia desmovilizados del conflicto armado en Colombia por parte de civiles y militares en uso de buen retiro, residentes en Bogotá
}

\author{
Civilian and military officials' attitudes toward demobilized population \\ of Colombian armed conflict, residents in Bogota, for a well retirement
}

Artículo de Investigación Copyright 2018 by Psicogente

\section{Correspondencia de autores:} karen.macias1004@gmail.com paulamendoza9723@hotmail.com chichanosorio97@gmail.com efriveros45@hotmail.com Ivera@usbbog.edu.co arlethpatriciab@gmail.com liliown@gmail.com

Recibido: 09-03-2017

Aceptado: 25-07-2017

Publicado: 01-01-18

\section{Karen Macías Pulgarín ${ }^{(D)}$, Paula Andrea Mendoza ${ }^{\circledR}$, Christian León Osorio ${ }^{D}$, Fernando Riveros Munévar ${ }^{\mathbb{D}}$, Anderssen Vera ${ }^{\mathbb{D}}$, Arleth Patricia Bernal ${ }^{\mathbb{D}}$ y Liliana Reyes ${ }^{(D)}$ Universidad de San Buenaventura, Bogotá, Colombia}

Resumen

Objetivo: Establecer las actitudes hacia los desmovilizados de la guerrilla por parte de civiles y militares en uso de buen retiro de Bogotá.

Método: El estudio tuvo un alcance descriptivo, con un método de comparación de grupos, se seleccionó una muestra de 120 participantes, bajo un procedimiento no probabilístico, con edades entre los 40 a 60 años distribuidos en 60 militares en uso de buen retiro y 60 civiles, residentes en la ciudad de Bogotá, a quienes se les aplicó el cuestionario de Actitudes hacia Desmovilizados de la Guerrilla.

Resultados: Se encontró que el 84,1 \% de los participantes presentan una actitud positiva o neutra, y estadísticos de comparación superiores al 0,05, lo que permite afirmar en discusión que ambos grupos presentan actitud positiva hacia los desmovilizados de la guerrilla, y que no se encuentran diferencias significativas entre civiles y militares en uso de buen retiro, residentes en Bogotá.

Conclusiones: Se afirma que el tener una actitud positiva, neutra o negativa no depende de la diferencia entre ser militar o civil.

Palabras Claves:

Militares, Civiles, Actitudes, Desmovilizados

Abstract

Objective: This study aims to establish civilian and military retired officials' attitudes towards guerrillas demobilization from Bogotá (Col), for a well retirement

Method: This study scope was descriptive, it was conducted, comparing groups, 120 selected participants, between 40 and 60 years, based on a not probabilistic procedure distributed as follows: 60 military retired officials and 60 civilians residents from Bogotá; a questionnaire related to attitudes toward demobilized population of armed conflict, was used.

Results: $84.1 \%$ of participants show a positive or neutral attitude, and statistical comparison higher than 0.05 ; this result, allows to say that both groups have a positive attitude towards demobilized guerrillas and there are not significant differences between civilian and military retired officials, residents in Bogotá.

Conclusion: As a conclusion, a positive, neutral or negative attitude does not depend on the difference between being military retired official or civilian.

Key words: Military, Civilians, Attitudes, Demobilized. 


\section{Karen Macías Pulgarín, Paula Andrea Mendoza, Christian León Osorio, Fernando Riveros Munévar, Anderssen Vera, Arleth Patricia Bernal, Liliana Reyes}

Como citar este artículo (APA):

Macías, K., Mendoza, P., León, C., Riveros, F., Vera, A., Bernal, A. \& Reyes, L. (2018). Actitudes hacia desmovilizados del conflicto armado en Colombia por parte de civiles y militares en uso de buen retiro, residentes en Bogotá. Psicogente, 21(39), 116-126. http://doi.org/10.17081/psico.21.39.2826

\section{INTRODUCCIÓN}

En Colombia se ha llevado a cabo uno de los conflictos armados más antiguos y con mayor número de muertes de la historia, según el informe general del Centro Nacional de Memoria Histórica (CNMH, 2013) se afirma que dicho conflicto ha traído consigo la muerte de aproximadamente 220.000 personas (civiles y militares) entre 1958 y 2012. Al respecto Saumeth (2010) plantea que las causas de las guerrillas colombianas son de origen tanto social como político, siendo así el resultado de dos procesos que se entrelazaron con fenómenos locales y regionales

Actualmente, como lo señala el Observatorio de procesos de Desarme, Desmovilización y Reintegración (ODDR) de la Universidad Nacional en 2011, estas guerrillas han ingresado en procesos de desmovilización, donde se da paso al desarme formal, a desmovilizaciones parciales, a intentos de reintegración a la vida social, política y económica, a partir de cuyos resultados y efectos se pueden afianzar las desmovilizaciones, para finalmente proceder a un desarme; siendo este último, el punto en que los integrantes de las organizaciones deciden desistir de las estructuras organizativas. Tal proceso lleva a observar diferentes aspectos que las involucran, ya que se reincorporarán a la vida civil, dentro de estos aspectos se destacan las actitudes que pueden tener personas externas a ellas, bien sean civiles, militares activos o en uso de buen retiro, entre otras poblaciones.

Sobre este aspecto la Agencia Colombiana para la Reintegración de personas y grupos alzados en armas o ACR (2016) refiere que dicho fin de las guerrillas implica un complejo proceso de desarme, desmovilización, reinserción y reintegración (DDRR) de excombatientes, consistente además en la decisión voluntaria e individual o colectiva de abandonar la pertenencia y el desarrollo de actividades en el grupo guerrillero. La ACR (2016) afirma que el DDRR es entendido como un proceso que coopera a la seguridad y estabilidad en una zona, finalizando con alguna situación de conflicto o violencia allí desarrolladas, desarmando a los combatientes y dotándolos de herramientas necesarias para la reintegración social y económica a la sociedad civil. Sobre este proceso, Patiño y Patiño (2012) indican que la reintegración es no solo la desmovilización, sino también un reacomodamiento subjetivo, una nueva articulación social en un contexto muchas veces hostil y sin las herramientas necesarias para afrontarlo.

Según Villegas (2016), los procesos de DDRR son un tema prioritario de políticas públicas en Colombia, donde se trabaja por el aumento en los índices de desmovilización individual y voluntaria por parte de los miembros de guerrillas, para esto se fortalecen campañas enfocadas a la prevención de reclutamiento, reconstrucción emocionalmente útil de sus experiencias y la funcionalidad del DDRR. Dadas las consecuencias del conflicto armado en el país, tales como desplazamiento, mortalidad, secuestros, extorsiones, violaciones y demás actos delictivos, es notorio que este ha 


\section{Karen Macías Pulgarín, Paula Andrea Mendoza, Christian León Osorio, Fernando Riveros Munévar, Anderssen Vera, Arleth Patricia Bernal, Liliana Reyes}

generado grandes daños a nivel físico, psicológico y social en toda la población colombiana, recayendo en desconfianza entre el desmovilizado y la sociedad, dicha desconfianza lleva consigo el escepticismo frente a capacidades de desmovilizados, sentimientos de rencor y venganza hacia ellos, mediado además por la misma experiencia que se haya tenido frente al conflicto, lo cual reitera la necesidad de finalizar el conflicto y realizar el proceso de DDRR (Fisas, 2011; Cortés, Acosta, Mora \& Gómez, 2013).

Tales factores psicológicos y sociales que pueden intervenir de manera favorable o desfavorable en alguna o todas las etapas del DDRR, se pueden abarcar en tres subescalas, que son la inclusión social, reincidencia y resiliencia, entendiendo la inclusión social (Subirats, Guillén \& Obradors, 2010) como el acto de proporcionar de un modo u otro, los recursos que se consideren convenientes para las personas o grupos que carecen de ellos, provocando cambios positivos en las dinámicas de desigualdad existentes.

Dentro de dichas desigualdades se encuentra la falta de confianza entre los desmovilizados y la sociedad, cuya posible causa según Aracena et al. (2000) puede ser el escepticismo por parte de la sociedad hacia la capacidad global que tiene el desmovilizado para mantener un funcionamiento efectivo frente a las adversidades del entorno, lo cual puede ser concerniente con el concepto de resiliencia. Por otro lado y teniendo en cuenta que según Roldán (2013) cualquier interferencia en tal capacidad de afrontamiento puede llevar a la reincidencia, esta se entiende como la repetición de actos delictivos por parte de los desmovilizados (Ossa, 2012).

Las condiciones anteriores retoman la necesidad de incluir en el proceso de reintegración, no solo a los mismos excombatientes y al gobierno, sino también a la misma sociedad civil como actores de la nueva construcción social (McEvoy, 2008; Shaw \& Waldorf, 2010; Gómez, 2017). De esta manera cabe mencionar a Villarraga (2013), quien indica que el éxito de tales procesos está relacionado con la debida atención de los derechos a la verdad, la justicia, la reparación de las víctimas y las garantías de no repetición de los graves hechos sucedidos.

Dadas las condiciones anteriores, la guerra ha generado una serie de actitudes tanto positivas como negativas de los colombianos hacia los guerrilleros y desmovilizados, definiéndose estas como una predisposición psicológica aprendida para responder o comportarse de forma favorable o desfavorable frente a un objeto o situación determinada (Kerlinger \& Lee, 2002, citados por Sulbarán, 2009; Morales, 2010; Riveros, Bohórquez, López \& Sepúlveda, 2015) y que contienen tres elementos: un componente cognitivo, relacionado con las opiniones o ideas de un individuo frente a la realidad, sin la estricta necesidad de conocer hechos probados u objetivamente ciertos acerca de esta; un componente afectivo, relacionado con la valoración positiva o negativa, favorable o desfavorable que cada persona siente respecto a algo; y un componente comportamental referido a lo que el sujeto hace, es decir, a la manifestación de conductas, frente a la situación o fenómeno.

En lo relacionado a actitudes hacia desmovilizados, la literatura es limitada, encontrándose estudios sobre creencias, percepciones y demás. Por ejemplo la investigación realizada por Montenegro (2015) donde se evaluó mediante entrevistas las percepciones de civiles frente al proceso de reintegración de desmovilizados en Bogotá, encontró que entre los desmovilizados y la sociedad 


\section{Karen Macías Pulgarín, Paula Andrea Mendoza, Christian León Osorio, Fernando Riveros Munévar, Anderssen Vera, Arleth Patricia Bernal, Liliana Reyes}

imperan las acciones de evitación a cualquier tipo de interacción o relación social entre sí, acompañadas de una desconfianza mutua generada por temor a poner en riesgo su integridad o la de sus familias.

Esta desconfianza también se vio reflejada en el estudio de Castaño (2015) donde por medio de entrevistas a civiles que fueron víctimas del conflicto con paramilitares, se identificó que los no combatientes (civiles) pertenecientes a poblaciones dominadas por paramilitares, mantenían los niveles de obediencia con respecto a los exparamilitares (desmovilizados), quienes a su vez son vistos como sujetos violentos y posibles delincuentes. Además, Castaño menciona que las conductas de rechazo hacia los desmovilizados se deben tanto al temor de la población, que si bien está inmerso en sus valores, como a los discursos socialmente reconocidos. Por otro lado, Roldán (2013) realizó un estudio evaluando la inclusión laboral de los desmovilizados, donde fue evidente la falta de voluntad social para acogerlos específicamente en dicho ámbito, lo cual según este mismo autor trae como consecuencia que decidan reincidir en la ilegalidad.

En este mismo sentido, Duque y Guevara (2014) afirman que por diversos sucesos se ha llegado a la idea de que el Estado colombiano no cuenta con la idoneidad para brindar programas y proyectos que permitan una verdadera incorporación de los desmovilizados a la sociedad civil, y que por tanto alrededor de los procesos de desmovilización y reintegración se encuentran diversas personas escépticas ante tales fenómenos.

Por otro lado, las actitudes han sido un ámbito altamente estudiado, sin embargo las percepciones de la sociedad frente a los desmovilizados y las actitudes hacia estos mismos, han sido limitados en cuanto a estudios cuantitativos o comparativos, por tanto se encuentra la necesidad de contrastar las actitudes de dos grupos posiblemente diferenciados, en su forma de percibir una población y que cuentan con prejuicios socialmente arraigados en Colombia. Esto, debido a que el contexto militar, así como lo señalan Loaiza y Posada (2016), es un entorno complejo debido a diversos factores, en especial los relacionados a su rígida estructura jerárquica, la cual lleva consigo un conjunto de valores y normas éticas que actúan como guías de conducta (tareas y funciones) para los militares siendo activos o retirados de las fuerzas militares de Colombia.

En este orden de ideas esta investigación tiene como objetivo principal establecer las actitudes hacia los desmovilizados de la guerrilla, por parte de civiles y militares en uso de buen retiro de Bogotá, teniendo en cuenta para la medición de las actitudes desde la inclusión social, la resiliencia y reincidencia relacionadas con el DDRR.

\section{MÉTODO}

\subsection{Diseño}

El presente es un estudio cuantitativo con alcance descriptivo por comparación de grupos (Hernández, Fernández \& Baptista, 2010) dado que se mide y compara una variable determinada en dos grupos diferentes, en este caso se midieron y compararon las actitudes de los militares en uso de buen retiro y civiles residentes en Bogotá, hacia los desmovilizados de la guerrilla. 


\section{Karen Macías Pulgarín, Paula Andrea Mendoza, Christian León Osorio, Fernando Riveros Munévar, Anderssen Vera, Arleth Patricia Bernal, Liliana Reyes}

\subsection{Participantes}

La muestra se seleccionó a través de un muestreo no probabilístico tipo bola de nieve, donde participaron 60 civiles y 60 militares en uso de buen retiro, de los cuales el 67,5\% eran hombres y el 32,5\% eran mujeres, las edades oscilaban entre los 40 y 60 años (media $=49,85$, d.e. $=5.752$ ), residentes en la ciudad de Bogotá, dentro de los criterios de inclusión, se tuvieron: la edad, la radicación mínima de tres años en Bogotá y que no fueran víctimas del conflicto armado por secuestro o desplazamiento, también se tuvo en cuenta que no tuvieran enfermedades cognitivas o neurológicas relevantes.

Cabe aclarar que el muestro por tipo bola de nieve en esta investigación fue necesario ya que el acceso a la muestra, en especial a los militares sean activos o en uso de buen retiro no es sencilla, de igual manera se tomó la decisión dentro de la investigación de utilizar el mismo tipo de muestro para la muestra civil.

\subsection{Instrumentos}

Se aplicó el cuestionario de Actitudes Hacia Desmovilizados de la Guerrilla, elaborado en el marco de la investigación (apéndice A), que mide las actitudes, distribuidas en cuatro subescalas (Inclusión, Resiliencia, Reincidencia, y total), y de acuerdo a sus componentes (afecto, cognición y comportamiento), consta de 30 afirmaciones en escala Likert, con cuatro opciones de respuesta (Muy de acuerdo, de acuerdo, en desacuerdo, muy en desacuerdo), donde 15 de estas afirmaciones son evaluadas de forma contraria en la puntuación, debido a que se refieren a la actitud negativa de los participantes. Se puede aplicar de forma grupal, tiene una duración aproximada de entre 20 a 30 minutos. La escala cuenta con validez de contenido, con puntuaciones entre 0,71 y 1 en sus subescalas, y con validez de constructo a partir del análisis factorial, y con un índice de consistencia interna con el alfa de Cronbach de 0,83.

\subsection{Procedimiento}

Se estableció inicialmente la fase contacto con un grupo de militares en uso de buen retiro radicados en la ciudad de Bogotá, los cuales facilitaron el contacto con el resto de la población militar, hasta completar el tamaño muestral requerido, quienes firmaron el consentimiento informado. Este mismo procedimiento se realizó para obtener la muestra de civiles. Posteriormente se realizó la fase de medición donde se procedió a la medición de la variable de actitudes, mediante el cuestionario Actitudes Hacia Desmovilizados de la Guerrilla en escala tipo Likert, el cual fue diligenciado en dos modalidades: 1) El cuestionario fue enviado por correo electrónico a cinco participantes militares en uso de buen retiro, los cuales se encargaron de reenviar el cuestionario una vez resuelto, en un tiempo límite de 10 días. 2) El cuestionario fue aplicado directamente a los militares restantes en uso de buen retiro y así mismo a los 60 civiles, en un área libre de distracciones para el investigador o para el participante. 


\section{Karen Macías Pulgarín, Paula Andrea Mendoza, Christian León Osorio, Fernando Riveros Munévar, Anderssen Vera, Arleth Patricia Bernal, Liliana Reyes}

\subsection{Análisis de los datos}

Los análisis se realizaron por medio del paquete SPSS versión 24 donde se evaluó mediante estadística descriptiva las medidas de tendencia central (media, desviación estándar, máximos y mínimos). Posteriormente se analizaron los datos por medio de estadística inferencial para determinar los niveles de normalidad en su distribución, encontrando normalidad en los puntajes generales, por lo cual la comparación entre los dos grupos se realizó empleando la prueba T para muestras independientes y un coeficiente de significancia de 0,05. En cuanto a las actitudes hacia los componentes del proceso de desmovilización (Inclusión, resiliencia, reincidencia), se encontró normalidad en el componente de inclusión por lo tanto se analizó por prueba $T$ para muestras independientes. Sin embargo, los componentes de resiliencia y reincidencia se analizaron mediante la prueba $U$ de Mann Whitney debido a la falta de normalidad en los datos.

\section{RESULTADOS}

Con el fin de mostrar los intervalos de interpretación del cuestionario, que permiten determinar el tipo de actitud según las puntuaciones, se anexa la Tabla 1.

Tabla 1

Intervalos de interpretación del cuestionario de actitudes.

\begin{tabular}{lccc}
\hline \multicolumn{1}{c}{ Subescala } & Positiva & Neutra & Negativa \\
\hline Inclusión & 33 a 44 & 23 a 32 & 11 a 22 \\
Resiliencia & 33 a 44 & 23 a 32 & 11 a 22 \\
Reincidencia & 24 a 32 & 18 a 23 & 8 a 17 \\
Total & 81 a 120 & 71 a 80 & 30 a 70 \\
\hline
\end{tabular}

Al respecto y teniendo en cuenta la tabla anterior, los datos muestran que el $50 \%$ del total de la muestra (30 civiles y 30 militares) tienen una actitud positiva, puesto que puntúan entre 81 y 120 en el puntaje general de la escala, el 34,4 \% (23 civiles y 18 militares) tienen una actitud neutra con puntuaciones entre 71 y 80 , y el 15,9\% (7 civiles y 12 militares) tienen una actitud negativa, es decir puntúan entre 30 y 70 en el puntaje general. Esto implica que tanto civiles como militares retirados tienen una actitud positiva hacia los desmovilizados de la guerrilla, sin embargo en el tipo de actitud neutra se encuentran más civiles que militares en contraparte con el tipo de actitud negativa donde se encuentran más militares que civiles (Figura 1). 


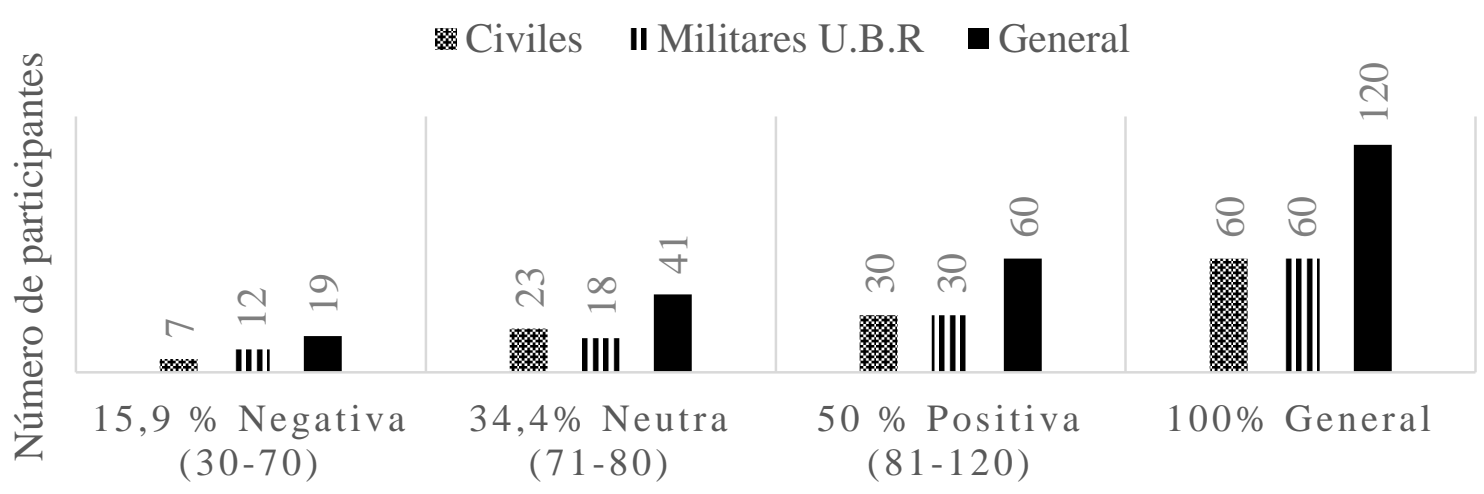

Tipo de actitud

Figura 1. Distribuciones del tipo de actitud en las dos muestras.

En lo que respecta a la comprobación de la hipótesis, como se observa en la Tabla 2, se realizó un análisis comparativo entre los grupos (civiles y militares en uso de buen retiro), inicialmente se comprobó la distribución normal, con la prueba Kolmogorov-Smirnov obteniendo normalidad en la actitud general (sig. > 0,200) y en el componente de inclusión (sig. 0,052), por lo tanto se analizaron los datos de estas variables usando la prueba t para muestras independientes, resultados mostrados en la Tabla 2.

Tabla 2

Prueba t para muestras independientes en las variables puntaje general y en inclusión.

\begin{tabular}{|c|c|c|c|}
\hline \multirow[t]{2}{*}{ Subescalas } & \multicolumn{2}{|c|}{ Medias } & \multirow{2}{*}{$\begin{array}{c}\text { Significancia } \\
\text { Unilateral }\end{array}$} \\
\hline & Civil & Militares U.B.R & \\
\hline AC General & 82,13 & 80,83 & 0,251 \\
\hline AC Inclusión & 29,98 & 30,05 & 0,476 \\
\hline
\end{tabular}

Nota. AC: Actitud, U.B.R: Uso de Buen Retiro.

Los datos de la Tabla 2 refieren que dada la significancia mayor a 0,05, se puede mencionar que no existen diferencias estadísticamente significativas en la actitud, ni a nivel general, ni en la subescala de inclusión, entre los civiles y militares en uso de buen retiro. En cuanto a los demás subescalas (resiliencia y reincidencia), al no evidenciar normalidad en sus datos, se realizó el análisis comparativo por medio de la prueba U de Mann Whitney. Los resultados se observan en la Tabla 3. 
Tabla 3.

Prueba U de Mann Whitney para las subescalas resiliencia y reincidencia.

\begin{tabular}{ccccc}
\hline Subescalas & \multicolumn{3}{c}{ Rango } & Significancia \\
\cline { 2 - 3 } & Civil & Militares U.B.R & Unilateral \\
AC Resiliencia & 64,48 & 56,53 & 0,1045 \\
AC Reincidencia & 61,92 & 59,08 & 0,326 \\
\hline Nota. AC: Actitud, U.B.R: Uso de Buen Retiro. & & &
\end{tabular}

Nota. AC: Actitud, U.B.R: Uso de Buen Retiro.

Finalmente, los datos hallados en la Tabla 3 refieren que, al arrojar las puntuaciones mayores al nivel del 0,05, indican que no existen diferencias estadísticamente significativas en las actitudes de civiles y militares en uso de buen retiro en las subescalas de resiliencia y de reincidencia.

\section{DISCUSIÓN}

Los impactos sociales, políticos y culturales que ha traído el conflicto armado en Colombia han generado una historia de incertidumbre hacia los procesos de paz y de desmovilización de las guerrillas, lo que recae en una controversia ideológica que poco ha sido estudiada, por lo cual resulta conveniente la realización de una investigación desde la realidad social, política y económica que atraviesa el país a causa de los procesos de acuerdos liderados por el Gobierno para la desmovilización y reintegración de la guerrilla, aspecto que hace relevante el presente estudio.

De acuerdo a los resultados obtenidos en la presente investigación, se puede afirmar que tanto civiles como militares en uso de buen retiro entre 40 y 60 años tienen una actitud positiva hacia los desmovilizados de la guerrilla y que no existen diferencias estadísticamente significativas entre estos dos grupos muestrales. Es decir, se evidencia una valoración positiva, junto con una manifestación de conductas y opiniones favorables hacia ellos, sin embargo aunque los grupos manifiesten una actitud positiva en el cuestionario, puede que exista una actitud neutra que no dirige la conducta, permitiendo que la persona modifique su actitud de acuerdo al contexto en el que se encuentra, refiriéndose al planteamiento de Aguilar (2011), un pensamiento "desapercibido" o prejuicio "sutil".

Debido a que no se encontró una diferencia significativa en la actitud de civiles y militares en uso de buen retiro entre 40 y 60 años, ni en el análisis de los resultados por las subescalas (inclusión, resiliencia y reincidencia), se plantea que el tener una actitud positiva, neutra o negativa no difiere del tipo de población a la que se pertenezca. Se resalta que a la luz de los resultados parece que hay una voluntad social por parte de los grupos evaluados hacia los desmovilizados de la guerrilla, es decir que no hay un escepticismo, sino más bien expectativas positivas hacia el desarme, desmovilización, reinserción y reintegración, contrario a investigaciones como la de Montenegro (2015), que refiere el escepticismo de la sociedad hacia los desmovilizados; la de Castaño (2015), 


\section{Karen Macías Pulgarín, Paula Andrea Mendoza, Christian León Osorio, Fernando Riveros Munévar, Anderssen Vera, Arleth Patricia Bernal, Liliana Reyes}

que refleja el temor y el rechazo a estos mismos y la de Roldán (2013), que evidencia la falta de voluntad social para acoger a esta población. Llama la atención también la actitud positiva de la muestra de militares en uso de buen retiro, puesto que va en contra de lo indicado por Gómez (2017), quien refería la negativa inicial al proceso de paz por parte de la Asociación Colombiana de Oficiales en Retiro (Acore).

Se considera que la causa de esto es que en el Gobierno actual se han implementado campañas por el Ministerio de Defensa (Villegas, 2016) para la prevención de reclutamiento y atención humanitaria a desmovilizados donde se utiliza la función comunicativa del lenguaje con la finalidad de cambiar el escepticismo de la sociedad hacia los desmovilizados de la guerrilla y generar actitudes positivas hacia ellos, lo que concuerda con Lucci (2006), quien afirma que el lenguaje permite la apropiación de la realidad, la interiorización de la cultura y la conformación de categorías, prejuicios y actitudes. Así mismo, tal como lo plantean Guerra y Plata (2005), el reto de una construcción de paz, es ante todo el de la construcción de una sociedad democrática. Sin embargo, para esto se debe analizar la experiencia de la sociedad estableciendo el adecuado balance entre las decisiones del Estado y la población, para así recuperar la vitalidad y la expresión de los actores.

De igual forma, las diferencias entre los estudios previos con el presente con respecto a la actitud de los militares retirados puede ser explicada por la vinculación reciente de un general en retiro de las mismas fuerzas armadas y del exdirector de la policía en el equipo negociador del Gobierno, así como la participación de militares activos en la subcomisión para el fin del conflicto (Cruz-Rodríguez, 2016).

Cabe resaltar que no se encuentran investigaciones válidas o confiables acerca de prejuicios, actitudes y percepciones de las fuerzas militares de Colombia hacia los desmovilizados y/o guerrilleros, por tanto este estudio que incluye una muestra de militares retirados puede llegar a ser un insumo inicial que permita guiar estudios posteriores acerca de las actitudes de miembros de las fuerzas militares hacia la desmovilización de las guerrillas colombianas.

Dado que los resultados de esta investigación permiten dar cuenta de que, si bien prima la actitud positiva, aún persiste una neutralidad y una actitud negativa hacia los desmovilizados a pesar del esfuerzo por parte del Gobierno y la ACR en el proceso de desarme, desmovilización, reinserción y reintegración (DDRR). Dado que el objetivo principal de estas organizaciones es dar fin al conflicto armado, se recalca que aún se tiene y se tendrá que trabajar más en los procesos que favorezcan la inclusión social de los desmovilizados como por ejemplo ayudas psicológicas a las familias y proporcionar recursos que se consideren convenientes para afrontar la desigualdad social, y especialmente el trabajo comunitario, pues como varios autores lo plantean (McEvoy, 2008; Shaw \& Waldorf, 2010; Gómez, 2017) es la sociedad civil uno de los máximos actores en este proceso de reintegración, y como lo refiere Cortés (2017) en su análisis del escrito de Ethel Nataly Castellanos, por supuesto es clave la vinculación en este proceso de reconciliación a las fuerzas armadas que también participaron en el conflicto armado. 


\section{Karen Macías Pulgarín, Paula Andrea Mendoza, Christian León Osorio, Fernando Riveros Munévar, Anderssen Vera, Arleth Patricia Bernal, Liliana Reyes}

Dentro de los aspectos a tener en cuenta para posteriores estudios, se sugiere ampliar la muestra, seleccionarla con procedimientos aleatorios, para que se puedan elaborar conclusiones más robustas, a partir de muestras representativas de la población objetivo. Ya que el poder de generalización queda limitado, lo que sustenta réplicas del presente estudio para corroborar estos resultados. A su vez se sugiere contar con más estudios del instrumento que permitan ampliar su uso y mejorar sus propiedades psicométricas. Una variable que no se tuvo en cuenta en este estudio fue el sexo de los participantes, dado que la muestra de militares retirados fue compuesta en su totalidad por hombres, y dado que el estudio llevado a cabo por Angulo, Ortiz y Pantoja (2014) sí refiere variaciones de las percepciones frente al proceso de paz con las FARC específicamente en torno a la desmovilización definitiva, la reconciliación con los miembros de las FARC y la participación política de los desmovilizados, se recomiendan investigaciones similares a la mostrada en el presente manuscrito, contemplando grupos de hombres y mujeres por aparte.

Teniendo en cuenta que hay escasas investigaciones sobre el tema de actitudes hacia los desmovilizados, se resalta que la presente investigación sirve de base para próximos estudios tanto cualitativos como cuantitativos que busquen relaciones entre las actitudes y distintos fenómenos políticos y culturales, por ejemplo campañas publicitarias, estrato socioeconómico y ubicación geográfica. Se sugiere replicar esta investigación haciendo un contacto previo con una organización que facilite el acceso a los militares en uso de buen retiro y con militares activos, además de mayores muestras en población civil, garantizando la generalización de los hallazgos acá encontrados.

Nota de Autores

El presente artículo es derivado de la investigación titulada "Actitudes hacia desmovilizados de la guerrilla por parte de militares en uso de buen retiro y civiles.

\section{REFERENCIAS}

Agencia Colombiana para la Reintegración de personas y grupos alzados en armas ACR (2016). Manual del sistema integrado de gestión para la reintegración-SIGER. Recuperado de http://www.reintegracion.gov.co/es/agencia/Documentos\%20de\%20Siger/2016\%201\%20Manua\% 20de\%20Sistema\%20Integrado\%20de\%20Gesti\%C3\%B3n\%20V4.pdf

Aguilar, J. (2011). Prejuicios, estereotipos y discriminación. México: Asociación Oaxaqueña de Psicología A.C.

Angulo, M., Ortiz, A. \& Pantoja, S. (2014). Análisis de las percepciones de los colombianos sobre el proceso de paz y el posconflicto desde una perspectiva de género. Colombia Internacional, 80(272), 220 233. http://dx.doi.org/10.7440/colombiaint80.2014.07

Aracena, M., Castillo, R., Haz, A., Cumsille, F., Muñoz, S., Bustos, L. \& Román, F. (2000). Resiliencia al maltrato físico infantil: variables que diferencian a los sujetos que maltratan y no maltratan físicamente a sus hijos en el presente y que tienen una historia de maltrato físico en la infancia. Revista de Psicología, 4(1), 11-28. DOI: 10.5354/0719-0581.2000.18543 


\section{Karen Macías Pulgarín, Paula Andrea Mendoza, Christian León Osorio, Fernando Riveros Munévar, Anderssen Vera, Arleth Patricia Bernal, Liliana Reyes}

Castaño, Z.D. (2015). Entre armas y razones: excombatientes y dominación en el postconflicto urbano en Moravia-Medellín/Colombia. Analecta Política, 5(8), 73-96. Dsiponible en https://revistas.upb.edu.co/index.php/analecta/article/view/3129

Centro Nacional de Memoria Histórica (CNMH). (2013). iBASTA YA! Colombia: Memorias de guerra y dignidad. Bogotá: Imprenta Nacional. Recuperado de https://www.centrodememoriahistorica.gov.co/descargas/informes2013/bastaYa/basta-yacolombia-memorias-de-guerra-y-dignidad-2016.pdf

Cortés, F. (2017). Justicia Transicional: memoria colectiva, reparación, justicia y democracia. Estudios SocioJurídicos, 19(1), 159-165. http://dx.doi.org/10.12804/revistas.urosario.edu.co/sociojuridicos/a.5288

Cortés, A., Acosta, A., Mora, J. \& Gómez, N. (2013). Desarme, Desmovilización y Reintegración, DDR: Una introducción para Colombia. Cuaderno de Análisis, 1(13), 1-88. Dsiponible en http://repository.unimilitar.edu.co/handle/10654/13498

Cruz-Rodríguez, E. (2016). Fuerza pública, negociaciones de paz y posacuerdo en Colombia. Bogotá: Desde Abajo.

Duque, L. \& Guevara, A. F. (2014). Las tensiones sociales producto de la desmovilización y reintegración de grupos guerrilleros $y \quad$ paramilitares. Recuperado de http://bibliotecadigital.usb.edu.co/bitstream/10819/2129/1/Las_Tensiones_Desmovilizacion_Gru pos_Paramilitares_Duque_2014.pdf

Fisas, V. (2011). Introducción al Desarme, Desmovilización y Reintegración (DDR) de excombatientes. Quaderns de Construcció de Pau, (24), 1-22. Dsiponible en http://escolapau.uab.es/img/qcp/introduccion_ddr.pdf

Gómez, G. (2017). Entre el castigo y la reconciliación. Análisis sociojurídico del proceso de paz y la negociación del Acuerdo sobre las Víctimas del Conflicto. Estudios Políticos, 50, 236-256. DOI: 10.17533/udea.espo.n50a13

Guerra, M. \& Plata, J. (2005). Estado de la investigación sobre conflicto, posconflicto, reconciliación y papel de la sociedad civil en Colombia. Revista de Estudios Sociales, 21, 81-92. Dsiponible en http://www.scielo.org.co/scielo.php?script=sci_arttext\&pid=S0123-885X2005000200008

Hernández, R., Fernández, C. \& Baptista, P. (2010). Metodología de la Investigación. México: McGraw-Hill.

Loaiza, O. \& Posada, J. L. (2016). Psicología militar: Conceptualización e investigaciones contemporáneas. PSIENCIA. Revista Latinoamericana de Ciencia Psicológica, 8(2), 1-20. DOI: 10.5872/psiencia/8.2.71

Lucci, M. (2006). La propuesta de Vygotsky: La Psicología Socio-histórica. Revista de curriculum y formación del profesorado, 10(2), 1-11. Dsiponible en http://www.ugr.es/ recfpro/rev102COL2.pdf

McEvoy, K. (2008). Letting Go of Legalism: Developing a "Thicker" Version of Transitional Justice. En: K. McEvoy \& L. McGregor (Eds.), Transitional Justice from Below. Grassroots Activism and the Struggle for Change. Portland: Hart. 


\section{Karen Macías Pulgarín, Paula Andrea Mendoza, Christian León Osorio, Fernando Riveros Munévar, Anderssen Vera, Arleth Patricia Bernal, Liliana Reyes}

Montenegro, C. (2015). Percepciones frente al proceso de reintegración de las y los desmovilizados en Bogotá: ¿Se está contribuyendo a una reconciliación social?. (Tesis de pregrado). Pontificia Universidad Javeriana, Bogotá, Colombia. Dsiponible en https://repository.javeriana.edu.co/handle/10554/17030

Morales, P. (2010). Guía para construir escalas de actitudes. Recuperado de http://blog.uca.edu.ni/dinorahmedrano/files/2011/08/Guiaparaconstruirescalasdeactitudes.pdf

Observatorio de procesos de Desarme, Desmovilización y Reintegración (ODDR) (2011). Desarme, Desmovilización y Reintegración (DDR), la complejidad de los procesos en Colombia. Recuperado de http://www.bdigital.unal.edu.co/8293/1/DDRComplejidadprocesosenColombia.pdf

Ossa, M. (2012). Aproximaciones conceptuales a la reincidencia penitenciaria. Revista Ratio Juris, 7(14), 113140. Dsiponible en http://publicaciones.unaula.edu.co/index.php/ratiojuris/article/view/143

Patiño, R. \& Patiño, C. (2012). Configuración de la identidad de desertores de la guerrilla colombiana. Psicologia \& Sociedade, 24(3), 517-526. Dsiponible en http://vufind.uniovi.es/Record/oai:doaj.orgarticle:0a90505840064b34982a2bb63af50bdb

Riveros, F., Bohórquez, D., López, S., \& Sepúlveda, E. (2015). Diseño y validación de un instrumento para medir las actitudes frente a la labor profesional del psicólogo. Revista Iberoamericana de psicología, ciencia y tecnología, 8(2), 55-66. Dsiponible en http://revistas.iberoamericana.edu.co/index.php/ripsicologia/article/view/814

Roldán, L. (2013). La inclusión laboral de los desmovilizados del conflicto en Colombia: Auténtico mecanismo emancipador de la violencia en Colombia. Universitas estudiantes, (10), 107-123. Dsiponible en http://cienciasjuridicas.javeriana.edu.co/documents/3722972/4350738/6+la+inclusi\%C3\%B3n+lab oral+107-124.pdf/6570fa55-76cb-4c55-8f37-06f5bf6764cb

Saumeth, E. (2010). Historia de la guerrilla en Colombia. Recuperado de http://ecsbdefesa.com.br/defesa/fts/HGC.pdf

Shaw, R. \& Waldorf, L. (2010). Localizing Transitional Justice. Interventions and Priorities after Mass Violence. Stanford: Stanford University.

Subirats, J., Guillén, E. \& Obradors, A. (2010). Ciudadanía e inclusión social frente a las inseguridades contemporáneas. La significación del empleo. Documentos de Trabajo (Fundación Carolina), 32(2010), 133-142. Dsiponible en http://www.academia.edu/2409263/CIUDADANIA_E_INCLUSI\%C3\%93N_SOCIAL_FRENTE_A_LAS_I NSEGURIDADES_CONTEMPOR\%C3\%81NEAS._LA_SIGNIFICACI\%C3\%93N_DEL_EMPLEO

Sulbarán, D. (2009). Medición de Actitudes. Recuperado de https://psicoexperimental.files.wordpress.com/2011/03/dimas-sulbaran-escalas-deactitudes1.Pdf

Villarraga, Á. (2013). Experiencias históricas recientes de reintegración de excombatientes en Colombia. Colombia internacional, (77), 107-140. http://dx.doi.org/10.7440/colombiaint77.2013.05 
Karen Macías Pulgarín, Paula Andrea Mendoza, Christian León Osorio, Fernando Riveros Munévar, Anderssen Vera, Arleth Patricia Bernal, Liliana Reyes

Villegas, L. (2016). Memorias al Congreso. Recuperado de https://www.mindefensa.gov.co/irj/go/km/docs/Mindefensa/Documentos/descargas/Documento s_Descargables/espanol/memorias2015-2016.pdf

Está obra está bajo: Creative commons attribution 4.0 international license. El beneficiario de la licencia tiene el derecho de copiar, distribuir, exhibir y representar la obra y hacer obras derivadas siempre y cuando reconozca y cite la obra de la forma especificada por el autor o el licenciante

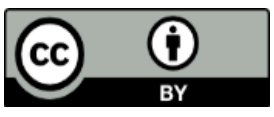

